\title{
FULL $C^{*}$-CROSSED PRODUCT DUALITY
}

\author{
JOHN C. QUIGG
}

(Received 26 April 1989; revised 12 October 1989)

Communicated by I. Raeburn

\begin{abstract}
Takai duality for full $C^{*}$-crossed products holds for twisted actions in the sense of Green and fails for coactions.
\end{abstract}

1980 Mathematics subject classification (Amer. Math. Soc.) (1985 Revision): 46 L 55.

\section{Introduction}

In [5], Katayama proved Takai duality for what we will call "reduced" crossed products of $C^{*}$-algebras by "reduced" coactions of locally compact groups. This involves regular representations of the crossed product and the dual crossed product, and the duality isomorphism is implemented by a unitary operator. In [12], Raeburn defined what we will call "full" coactions and corresponding "full" crossed products, and proved the appropriate version of Takai duality. This involves the full $C^{*}$-algebra of the group, and the theory has a more categorical flavor than in the reduced case. In a little more detail (refer to the next section for definitions), if $A$ is a $C^{*}$-algebra carrying an automorphic action of a locally compact group $G$, then Raeburn defines a dual (full) coaction of $G$ on the (full) crossed product $A \times G$ and proves that the dual (full) crossed product $A \times G \times \widehat{G}$ is isomorphic to the tensor product $A \otimes \mathscr{K}$, where $\mathscr{K}$ denotes the $C^{*}$-algebra of compact operators on the Hilbert space $L^{2}(G)$. He leaves open the question whether this result

(C) 1991 Australian Mathematical Society $0263-6115 / 91 \$ A 2.00+0.00$ 
extends to twisted actions, which we answer in the affirmative (Theorem 3.6) for the case of twisted actions in Green's sense. He also shows that if $A$ carries a coaction of $G$, then there is natural way to define a dual action of $G$ on the crossed product $A \times \widehat{G}$, and asks whether the dual crossed product $A \times \widehat{G} \times G$ is isomorphic to $A \otimes \mathscr{K}$. We answer this in the negative (Example 2.12); roughly speaking, what "goes wrong" is that crossed products by coactions are automatically reduced.

A comprehensive reference for $C^{*}$-algebras, locally compact groups, and $C^{*}$-crossed products by actions is [10].

Part of the research for this paper was carried out while the author was visiting the mathematics department at the University of Georgia, and he would like to thank his hosts, especially Elliot Gootman, for their hospitality.

\section{Full coactions}

We first establish some notation and recall some of the definitions of [12]. Let $A$ be a $C^{*}$-algebra and let $M(A)$ denote its multiplier algebra. If $B$ is another $C^{*}$-algebra, we call a homomorphism $\pi: A \rightarrow M(B)$ "strict" if for every bounded approximate identity $\left\{e_{i}\right\}$ of $A$ the net $\left\{\pi\left(e_{i}\right)\right\}$ converges strictly to 1 . Recall that for $C^{*}$-algebras $A$ and $B$

$$
\widetilde{M}(A \otimes B)=\{m \in M(A \otimes B): m(1 \otimes B) \cup(1 \otimes B) m \subset A \otimes B\},
$$

where $\otimes$ denotes the minimal $C^{*}$-tensor product. Let $G$ be a locally compact group. Let $C^{*}(G)$ and $C_{r}^{*}(G)$ denote the full and the reduced $C^{*}$ algebras of $G$, respectively, let $U_{G}, \lambda$, and $\rho$ denote the universal, left regular, and right regular representations of $G$, respectively, and let $A(G)$ and $B(G)$ denote the Fourier and the Fourier-Stieltjes algebras of $G$, respectively.

Definition 2.1. A full coaction of $G$ on $A$ is a strict monomorphism $\delta: A \rightarrow \widetilde{M}\left(A \otimes C^{*}(G)\right)$ such that

$$
\delta \otimes \imath \circ \delta=\imath \otimes \delta^{G} \circ \delta,
$$

where $\delta^{G}: C^{*}(G) \rightarrow \widetilde{M}\left(C^{*}(G) \otimes C^{*}(G)\right)$ is the homomorphism determined by

$$
\delta^{G}\left(U_{G}(s)\right)=U_{G}(s) \otimes U_{G}(s), \quad s \in G .
$$

For $\phi \in B(G)$ we use $\delta_{\phi}$ to denote $S_{\phi} \circ \delta$, where $S_{\phi}$ is the slice map from $A \otimes C^{*}(G)$, hence $\widetilde{M}\left(A \otimes C^{*}(G)\right.$ ) (see [6, Lemma 1.5]) to $A$. Although in [12] Raeburn does not require $\delta$ to be injective, we have no need of this extra generality. However, as Raeburn points out, if $\delta$ were not injective then $A$ would be the semidirect product (not $C^{*}$-algebraic direct sum, as 
stated in [12]) of the ideal $I=\operatorname{ker} \delta$ and the $C^{*}$-subalgebra $B=\delta_{1}(A)$. For example, if $A$ is such a semidirect product, one can define a "noninjective coaction" of $G$ on $A$ by

$$
\delta(x+b)=b \otimes 1, \quad x \in I, b \in B .
$$

Since we require $\delta$ to be faithful, we have $\delta_{1}=l_{A}$, the identity map on $A$. Another slight departure from Raeburn's definition is that he uses the maximal $C^{*}$-tensor product. However, if $\delta$ is an injective coaction in Raeburn's sense, then $\delta_{1}=t_{A}$ still holds, so that $\delta(A)$ has zero intersection with the annihilator of $A^{*} \otimes B(G)$, whence the composition formed by following $\delta$ by the canonical map into the minimal $C^{*}$-tensor product (extended naturally to the multiplier algebras) remains injective. Moreover, the covariant representations (which we will define presently) are the same with either choice of tensor product. Consequently, we saw no penalty to be incurred through the use of the more manageable minimal $C^{*}$-tensor product. On the other hand, it is not clear whether every coaction, as we have defined the term, factors through the maximal $C^{*}$-tensor product, and the maximal $C^{*}$ tensor product could conceivably be useful for some purposes (for example, investigation of ideals, perhaps), so it may prove convenient to return to the maximal $C^{*}$-tensor product at some other time.

We use the term "full" to distinguish the present type of coaction from the more familiar version defined in, for example, $[5,6]$, which uses the reduced rather than the full $C^{*}$-algebra of $G$. We call this latter type of coaction "reduced", and use the term "coaction" with no modifier to mean "full coaction". As Katayama [5] points out, if $\delta$ is a coaction, then $l \otimes \lambda \circ \delta$ factors through a reduced coaction on a quotient of $A$. Observe that $\delta^{G}$ is a coaction of $G$ on $C^{*}(G)$, and $\imath \otimes \lambda \circ \delta^{G}$ factors through the usual reduced coaction on $C_{r}^{*}(G)$.

Definition 2.2. $\delta$ is called nondegenerate if $\delta_{A(G)}(A)$ is dense in $A$.

If $\delta$ is nondegenerate, then so is the corresponding reduced coaction (on the appropriate quotient of $A$ ). Katayama [5, Proposition 7] states that full coactions are automatically nondegenerate, but we have been unable to understand his proof. It is not hard to verify that $\delta^{G}$ is nondegenerate. Also, [5, Lemma 3] can be adapted to show that if $\delta$ is nondegenerate then $\delta(A)\left(1 \otimes C^{*}(G)\right)$ is dense in $A \otimes C^{*}(G)$. Unless otherwise stated we shall assume henceforth that all coactions are nondegenerate.

We follow Raeburn in using $W_{G}$ to denote the universal representation of $G$, regarded as a unitary element of $M\left(C_{0}(G) \otimes C^{*}(G)\right)$. If $W$ is a representation of $C_{0}(G)$ we also let $W$ denote the unitary element $W \otimes l\left(W_{G}\right)$ of $M\left(W\left(C_{0}(G)\right) \otimes C^{*}(G)\right)$. The unitary $l \otimes \lambda(W)$ is a "corepresentation" in the sense of [6, Remark 3.2(2)], and conversely $W$ is uniquely determined 
by this corepresentation. Recall the slice map property

$$
W(\phi)=S_{\phi}(W), \quad \phi \in B(G) .
$$

Raeburn's definition of covariance and crossed product can be stated as follows.

Definition 2.3. (i) A covariant representation of $(A, G, \delta)$ is a pair $(\pi, W)$ of nondegenerate representations of $A$ and $C_{0}(G)$, respectively, such that

$$
W \in \operatorname{Hom}(\pi \otimes 1, \pi \otimes 1 \circ \delta),
$$

the set of intertwining operators for the representations $\pi \otimes 1$ and $\pi \otimes 1 \circ \delta$ of $A$ on $\mathscr{H} \otimes \mathscr{X}_{G}$, where $\mathscr{H}$ is the Hilbert space of $(\pi, W)$ and $\mathscr{K}_{G}$ is the space of $U_{G}$.

(ii) For a covariant representation $(\pi, W)$ of $(A, G, \delta)$ we define

$$
C^{*}(\pi, W)=C^{*}\left[\pi(A) W\left(C_{0}(G)\right)\right],
$$

where $C^{*}[S]$ denotes the $C^{*}$-algebra generated by the set $S$. It can be shown as in [6] that

$$
\pi(A) \cup W\left(C_{0}(G)\right) \subset M\left(C^{*}(\pi, W)\right) .
$$

(iii) A covariant representation $(\pi, W)$ is said to be weakly contained in another covariant representation $(\sigma, V)$ if there is a representation $\nu$ of $C^{*}(\sigma, V)$ such that

$$
\nu \circ \sigma=\pi, \quad \nu \circ V=W .
$$

We call $(\pi, W)$ and $(\sigma, V)$ weakly equivalent if each weakly contains the other.

(iv) A covariant representation is called weakly universal if it weakly contains every covariant representation. Of course, a weakly universal covariant representation is unique up to weak equivalence.

(v) The crossed product of $A$ by the coaction $\delta$ of $G$ is

$$
A \times_{\delta} \widehat{G}=C^{*}\left(\pi_{\delta}, W_{\delta}\right),
$$

where $\left(\pi_{\delta}, W_{\delta}\right)$ will denote a weakly universal covariant representation of $(A, G, \delta)$. We write $A \times \widehat{G}$ when $\delta$ is understood. Of course, the isomorphism class of the crossed product is independent of the choice of the weakly universal covariant representation. For a covariant representation $(\pi, W)$ of $(A, G, \delta)$ we denote the corresponding representation of $A \times \widehat{G}$ by $\pi \times W$.

If $(\pi, W)$ is a covariant representation of $(A, G, \delta)$ in the above sense, then $(\pi, l \otimes \lambda(W))$ is a covariant pair of representations of $(A, G, \delta)$ in the sense of [6, Definition 3.5], and conversely $(\pi, W)$ is uniquely determined by this covariant pair. 
Proposition 2.4. If $(\pi, W)$ is a covariant representation of $(A, G, \delta)$, then

$$
\delta^{W}(x)=\operatorname{Ad} W(x \otimes 1)
$$

defines a coaction of $G$ on $\pi(A)$. Moreover, $\delta^{W}$ is nondegenerate if $\delta$ is.

Proof. One verifies routinely that $\delta^{W}$ is a coaction on $\pi(A)$, and nondegeneracy then follows from the easily established identity

$$
\delta_{\phi}^{W} \circ \pi=\pi \circ \delta_{\phi}, \quad \phi \in B(G) .
$$

The covariance condition for $(\pi, W)$ may alternatively be written

$$
\delta^{W} \circ \pi=\pi \otimes \imath \circ \delta .
$$

Let $\alpha^{G}$ denote the action of $G$ on $C_{0}(G)$ by left translation, that is,

$$
\alpha_{s}^{G}(\phi)(t)=\phi\left(s^{-1} t\right), \quad \phi \in C_{0}(G), s, t \in G .
$$

Recall that a $C^{*}$-algebra $B$ acts to the right on its dual by

$$
\omega \cdot b(c)=\omega(b c), \quad \omega \in B^{*}, b, c \in B .
$$

Proposition 2.5. If $U$ and $W$ are representations of $G$ and $C_{0}(G)$, respectively, on the same Hilbert space, then $(U, W)$ is a covariant representation of $\left(C^{*}(G), G, \delta^{G}\right)$ if and only if $(W, U)$ is a covariant representation of $\left(C_{0}(G), G, \alpha^{G}\right)$.

Proof. Assume that $(U, W)$ is a covariant representation of $\left(C^{*}(G)\right.$, $\left.G, \delta^{G}\right)$. Then for $\phi \in B(G), s \in G$

$$
\begin{aligned}
W(\phi) U(s) & =S_{\phi}(W) U(s)=S_{\phi}(W(U(s) \otimes 1))=S_{\phi}\left(\delta^{W} \circ U(s) W\right) \\
& =S_{\phi}\left(\left(U(s) \otimes U_{G}(s)\right) W\right)=U(s) S_{\phi \cdot U_{G}(s)}(W) \\
& =U(s) W\left(\phi \cdot U_{G}(s)\right)=U(s) W \circ \alpha_{s^{-1}}^{G}(\phi),
\end{aligned}
$$

so that $(W, U)$ is a covariant representation of $\left(C_{0}(G), G, \alpha^{G}\right)$. The above argument can clearly be reversed, so we are done.

Let $\mathscr{K}(\mathscr{H})$ denote the $C^{*}$-algebra of compact operators on the Hilbert space $\mathscr{H}$, and let $\mathscr{K}$ by itself denote $\mathscr{K}\left(L^{2}(G)\right)$. Let $M$ denote the representation of $C_{0}(G)$ on $L^{2}(G)$ by multiplication.

COROLlARY 2.6. $(\lambda, M)$ is a weakly universal covariant representation of $\left(C^{*}(G), G, \delta^{G}\right)$, and

$$
\mathscr{K}=C^{*}(G) \times_{\delta^{G}} \widehat{G}
$$


Proof. Recall that every covariant representation of $\left(C_{0}(G), G, \alpha^{G}\right)$ is of the form $(1 \otimes M, 1 \otimes \lambda)$, and that

$$
\mathscr{K}=C^{*}\left[C_{r}^{*}(G) C_{0}(G)\right] .
$$

The above result shows that a weakly universal covariant representation $\left(\pi_{\delta}, W_{\delta}\right)$ of $(A, G, \delta)$ is not necessarily faithful on $A$. However, by Proposition $2.4, \delta^{W_{\delta}}$ is a coaction of $G$ on $\pi_{\delta}(A)$, and clearly $(A, G, \delta)$ and $\left(\pi_{\delta}(A), G, \delta^{W_{\delta}}\right)$ have (essentially) the same covariant representations and therefore the same crossed product. Of course, $\left(l, W_{\delta}\right)$ is a weakly universal covariant representation of $\left(\pi_{\delta}(A), G, \delta^{W_{\delta}}\right)$. As we shall see, $W_{\delta}$ is always faithful on $C_{0}(G)$.

Part (i) of the following definition is a routine adaptation of [2, Definition 2.2] to the context of full coactions.

DEFINITION 2.7. (i) If $\pi$ is a representation of $A$ on $\mathscr{H}$, then the induced covariant representation, or covariant representation induced from $\pi$, is the covariant representation of $(A, G, \delta)$ on $\mathscr{H} \otimes L^{2}(G)$ defined by

$$
\left(\tilde{\pi}, W_{\pi}\right)=(\pi \otimes \lambda \circ \delta, 1 \otimes M) .
$$

(ii) The regular covariant representation of $(A, G, \delta)$ is the covariant representation induced from any faithful representation of $A$; it is easily checked to be unique up to weak equivalence.

(iii) The reduced crossed product of $A$ by $\delta$ is

$$
A \times_{\delta, r} \widehat{G}=C^{*}\left(\tilde{\pi}, W_{\pi}\right),
$$

where $\pi$ is any faithful representation of $A$. As usual, the isomorphism class of the reduced crossed product is uniquely determined.

Proposition 2.8. (i) The regular covariant representation of $(A, G, \delta)$ is weakly universal, so that

$$
A \times_{\delta} \widehat{G}=A \times_{\delta, r} \widehat{G} .
$$

(ii) $l \otimes \lambda \circ \delta^{W_{\delta}}$ is a reduced coaction of $G$ on $\pi_{\delta}(A)$, and the associated crossed product as defined in $[5,6]$ agrees with $A \times_{\delta} \widehat{G}$.

Proof. (i) In view of the remark following Definition 2.3, this follows immediately from [6, Theorem 3.7].

(ii) By part (i) and Proposition 2.4 this is essentially [5, Proposition 7] (minus the assertion regarding nondegeneracy—see the comment following Definition 2.2). 
Observe that the reduced coaction $l \otimes \lambda \circ \delta^{W_{\delta}}$ of $G$ on $\pi_{\delta}(A)$ lifts to the full coaction $\delta^{W_{\delta}}$. We do not know if every reduced coaction lifts to a full coaction.

Let $\beta^{G}$ denote the action of $G$ on $C_{0}(G)$ by right translation, that is,

$$
\beta_{s}^{G}(\phi)(t)=\phi(t s), \quad \phi \in C_{0}(G), s, t \in G .
$$

The following definition is taken from [12].

Definition 2.9. The dual action $\widehat{\delta}$ of $G$ on $A \times \widehat{G}$ is determined by

$$
\widehat{\delta}_{s} \circ \pi_{\delta}=\pi_{\delta}, \quad \widehat{\delta}_{s} \circ W_{\delta}=W_{\delta} \circ \beta_{s}^{G}, \quad s \in G .
$$

When $A \times \widehat{G}$ is identified with the crossed product of $\pi_{\delta}(A)$ by the reduced coaction $l \otimes \lambda \circ \delta^{W_{\delta}}$ (Proposition 2.8(ii)), it is readily verified that $\widehat{\delta}$ agrees with the dual action of Katayama [5]. Therefore, the following result is just a restatement of Katayama's duality theorem [5, Theorem 8] (except that we abstain from writing down the double dual coaction).

THEOREM 2.10. $A \times \widehat{G} \times, G \cong \pi_{\delta}(A) \otimes \mathscr{K}$.

COROLlary 2.11. A representation of $A \times \widehat{G}$ is (equivalent to one) induced from a representation of $A$ if and only if it extends to a representation of $A \times \widehat{G} \times, G$.

Proof. Thanks to Theorem 2.10 this follows almost immediately from [2, Proposition 2.11 and Corollary 2.12].

The above result is an imprimitivity theorem, and it is a special case of a result of Mansfield [7, Theorem 28], although he uses reduced coactions and his dual action is by an amenable group. We point out that Theorem 2.10 can be derived from Corollary 2.11. To see this, first replace (temporarily) $A$ by $\pi_{\delta}(A)$, so that we need to show

$$
A \times \widehat{G} \times{ }_{r} G \cong A \otimes \mathscr{K} .
$$

Let $\pi \times W \times U$ be a faithful representation of $A \times \widehat{G} \times, G$. By the imprimitivity theorem there is a representation $\sigma$ of $A$ such that $\pi \times W \cong \tilde{\sigma} \times W_{\sigma}$, and it follows from Mansfield's proof of the imprimitivity theorem (which shows [7, Proposition 26] that $A \times \widehat{G} \times{ }_{r} G$ is the imprimitivity algebra in Rieffel's sense [13] for induction from $A$ to $A \times \widehat{G}$ ) that the same equivalence takes $U$ to $1 \otimes \rho$. Since $\delta$ is nondegenerate, it follows from [5, Theorem 5] that

$$
C^{*}\left(\tilde{\sigma} \times W_{\sigma}, 1 \otimes \rho\right)=\sigma(A) \otimes \mathscr{K} .
$$

Here we use the notation

$$
C^{*}(\pi, U)=C^{*}\left[\pi(B) U\left(C^{*}(G)\right)\right]
$$


when $(\pi, U)$ is a covariant representation of a system $(B, G, \alpha)$, with $\alpha$ an action of $G$ on a $C^{*}$-algebra $B$. Finally, it again follows from Mansfield's proof of the imprimitivity theorem that $\sigma$ is faithful since $\pi \times W \times U$ is faithful.

Theorem 2.10 can be used to show that full $C^{*}$-crossed product duality for coactions fails, answering in the negative a question left open by Raeburn in [12]. Specifically, full duality would require

$$
A \times \widehat{G} \times G \cong A \otimes \mathscr{K} ;
$$

even when $A$ has been replaced by a suitable quotient, allowing it to be faithfully embedded in the multiplier algebra of the crossed product, Theorem 2.10 shows that full duality will tend to fail to the extent that the full and reduced double crossed products may differ. We illustrate this phenomenon with an example.

EXAMPLE 2.12. We modify the canonical coaction on the $C^{*}$-algebra of $G$ to get a full coaction on the reduced $C^{*}$-algebra:

$$
\delta(\lambda(s))=\lambda(s) \otimes U_{G}(s), \quad s \in G .
$$

By Corollary 2.6 this coaction has the same covariant representations as $\delta^{G}$, so

$$
C_{r}^{*}(G) \times \widehat{G}=C^{*}(G) \times \widehat{G}=\mathscr{K} .
$$

Moreover, it is easy to see that the dual action on $\mathscr{K}$ is Ad $\rho$. This action is implemented by a representation in $M(\mathscr{K})=\mathscr{L}\left(L^{2}(G)\right)$ and is therefore outer conjugate to the trivial action, so

$$
C_{r}^{*}(G) \times \widehat{G} \times G=\mathscr{K} \times G=\mathscr{K} \otimes C^{*}(G),
$$

which is not isomorphic to $C_{r}^{*}(G) \otimes \mathscr{K}$ in general, for example, when $G$ is the free group on two generators. We will say more about failure of full duality toward the end of Section 3.

\section{Twisted actions}

We extend Raeburn's duality theorem for full crossed products by actions [12, Theorem 7] to the twisted crossed products of Green [3], at least in the presence of a measurable cross section. Let $\alpha$ be an action of $G$ on $A$, let $N$ be a closed normal subgroup of $G$, and let $\tau$ be a strictly continuous unitary representation of $N$ in $M(A)$ such that

$$
\alpha \mid N=\operatorname{Ad} \tau, \quad \alpha_{s} \circ \tau=\tau \circ \operatorname{Ad} s \quad(s \in G) .
$$

Modifying Green's notation and terminology slightly, we call $(\alpha, \tau)$ a twisted action of $G$ on $A$, define a covariant representation of $(A, G, \alpha, \tau)$ to be 
a covariant representation $(\pi, U)$ of $(A, G, \alpha)$ which preserves $\tau$ in the sense that $\pi \circ \tau=U \mid N$, and further define the twisted crossed product of $A$ by $(\alpha, \tau)$ to be

$$
A \tilde{\times}_{\tau} G=C^{*}\left(\pi_{\tau}, U_{\tau}\right)
$$

for a weakly universal covariant representation $\left(\pi_{\tau}, U_{\tau}\right)$ of $(A, G, \alpha, \tau)$. We write $A \tilde{\times} G$ when $\tau$ is understood.

Proposition 3.1. There is a nondegenerate coaction $\widehat{\alpha}$ of $G / N$ on $A \tilde{\times} G$ determined by

$$
\widehat{\alpha} \circ \pi_{\tau}=\pi_{\tau} \otimes 1, \quad \hat{\alpha} \circ U_{\tau}=U_{\tau} \otimes U_{G / N}
$$

Proof. The above formulae clearly give a covariant representation of $(A, G, \alpha, \tau)$.

We show that

$$
\widehat{\alpha}(A \widetilde{\times} G) \subset \widetilde{M}\left(A \widetilde{\times} G \otimes C^{*}(G / N)\right) .
$$

Let $a \in A, f \in C_{c}(G), g \in C_{c}(G / N)$. Then

$$
\begin{aligned}
\widehat{\alpha}\left(\pi_{\tau}(a) U_{\tau}(f)\right)\left(1 \otimes U_{G / N}(g)\right) \\
=\left(\pi_{\tau}(a) \otimes 1\right) \int_{G} f(s)\left(U_{\tau}(s) \otimes U_{G / N}(s N)\right) d s \\
\quad \times \int_{G / N} g(t N)\left(1 \otimes U_{G / N}(t N)\right) d(t N) \\
=\left(\pi_{\tau}(a) \otimes 1\right) \int_{G} \int_{G / N} f(s) g(t N)\left(U_{\tau}(s) \otimes U_{G / N}(s t N)\right) d(t N) d s \\
=\left(\pi_{\tau}(a) \otimes 1\right) \int_{G} \int_{G / N} f(s) g\left(s^{-1} t N\right)\left(U_{\tau}(s) \otimes U_{G / N}(t N)\right) d(t N) d s \\
=\left(\pi_{\tau}(a) \otimes 1\right) \int_{G} \int_{G / N} \sum_{i=1}^{n} f_{i}(s) g_{i}(t N)\left(U_{\tau}(s) \otimes U_{G / N}(t N)\right) d(t N) d s
\end{aligned}
$$

(approximate $(s, t N) \mapsto f(s) g\left(s^{-1} t N\right)$ in the inductive limit topology of $C_{c}(G \times G / N)$ by $\left.\sum f_{i} \otimes g_{i}\right)$

$$
\begin{aligned}
& =\sum_{i=1}^{n} \int_{G} \pi_{\tau}(a) f_{i}(s) U_{\tau}(s) d s \otimes \int_{G / N} g_{i}(t N) U_{G / N}(t N) d(t N) \\
& =\sum_{i=1}^{n} \pi_{\tau}(a) U_{\tau}\left(f_{i}\right) \otimes U_{G / N}\left(g_{i}\right) \\
& \in A \tilde{\times} G \otimes C^{*}(G / N),
\end{aligned}
$$


and similarly for

$$
\left(1 \otimes U_{G / N}(g)\right) \hat{\alpha}\left(\pi_{\tau}(a) U_{\tau}(f)\right) .
$$

It is obvious that $\widehat{\alpha}$ is a strict monomorphism, the injectivity following from the weak equivalence of the covariant representations $\left(\pi_{t}, U_{\tau}\right)$ and $\left(\hat{\alpha} \circ \pi_{\tau}, \hat{\alpha} \circ U_{\tau}\right)$. The coaction identity

$$
\widehat{\alpha} \otimes \imath \circ \widehat{\alpha}=\imath \otimes \delta^{G / N} \circ \widehat{\alpha}
$$

is readily checked on the generators.

Finally, the nondegeneracy follows from

$$
\widehat{\alpha}_{\phi}\left(\pi_{\tau}(a) U_{\tau}(f)\right)=\pi_{\tau}(a) U_{\tau}(\phi f),
$$

for $\phi \in A(G / N), a \in A, f \in L^{1}(G)$, where $\phi$ has been lifted to a continuous bounded function on $G$.

For a representation $\pi$ of $A$ on $\mathscr{H}$ (one formulation of) Green's induced covariant representation $\left(\tilde{\pi}, U_{\pi}\right)$ of $(A, G, \alpha, \tau)$ acts on the Hilbert space $\widetilde{\mathscr{H}}$ of measurable maps $\xi: G \rightarrow \mathscr{H}$ satisfying

$$
\xi(s n)=\pi \circ \tau\left(n^{-1}\right) \xi(s), \quad s \in G, n \in N,
$$

and

$$
\int_{G / N}\|\xi(s)\|^{2} d(s N)<\infty
$$

and it is defined by the formulae

$$
[\tilde{\pi}(a) \xi](t)=\pi \circ \alpha_{t^{-1}}(a) \xi(t), \quad\left[U_{\pi}(s) \xi\right](t)=\xi\left(s^{-1} t\right),
$$

for $a \in A, \xi \in \widetilde{\mathscr{H}}, s, t \in G$. Define a representation $W_{\pi}$ of $C_{0}(G / N)$ on $\widetilde{\mathscr{H}}$ by

$$
\left[W_{\pi}(\phi) \xi\right](t)=\phi(t N) \xi(t),
$$

for $\phi \in C_{0}(G / N), \underset{\tilde{x}}{\xi} \in \mathscr{H}, t \in G$. That $\left(\tilde{\pi} \times U_{\pi}, W_{\pi}\right)$ is a covariant representation of $(A \widetilde{\times} G, G / N, \widehat{\alpha})$ follows from the following result.

Proposition 3.2. Let $(\pi, U)$ be a covariant representation of $(A, G, \alpha, \tau)$, and let $W$ be a representation of $C_{0}(G / N)$ on the same Hilbert space. Then $(\pi \times U, W)$ is a covariant representation of $(A \widetilde{\times} G, G / N, \widehat{\alpha})$ if and only if $\pi$ commutes with $W$ and

$$
\operatorname{Ad} U(s) \circ W=W \circ \alpha_{s N}^{G / N}, \quad s \in G
$$

Proof. This is a routine computation using slice map techniques, and is similar to [8, pages $194-195]$. 
Green defines a twisted action $(\beta, \kappa)$ of $G$ on $A \otimes C_{0}(G / N)$ by

$$
\beta_{s}=\alpha_{s} \otimes \alpha_{s N}^{G / N}, \quad \kappa=\tau \otimes 1,
$$

and shows that $\left(A \otimes C_{0}(G / N)\right) \tilde{\times} G$ is the imprimitivity algebra for induction from $A$ to $(A, G, \alpha, \tau)$. Because of this the crux of our duality theorem is the following result. Let $\left(\pi_{\widehat{\alpha}} \times U_{\widehat{\alpha}}, W_{\widehat{\alpha}}\right)$ and $\left(\pi_{\kappa} \times W_{\kappa}, U_{\kappa}\right)$ be weakly universal covariant representations of $(A \widetilde{\times} G, G / N, \widehat{\alpha})$ and $(A \otimes$ $\left.C_{0}(G / N), G, \beta, \kappa\right)$, respectively.

Proposition 3.3. $\left(\pi_{\kappa} \times U_{\kappa}, W_{\kappa}\right)$ is a covariant representation of $(A \tilde{\times} G$, $G / N, \widehat{\alpha})$, and determines an isomorphism of $A \tilde{\times} G \times(G / N)^{-}$with

$$
\left(A \otimes C_{0}(G / N)\right) \tilde{\times} G .
$$

Proof. The first statement is readily verified, and the second can be seen by checking that $\left(\pi_{\widehat{\alpha}} \times W_{\widehat{\alpha}}, U_{\widehat{\alpha}}\right)$ is a covariant representation of $\left(A \otimes C_{0}(G / N)\right.$, $G, \beta, \kappa)$ which determines an inverse map for $\left(\pi_{\kappa} \times U_{\kappa}, W_{\kappa}\right)$.

COROllary 3.4. Every covariant representation of $(A \tilde{x} G, G / N, \widehat{\alpha})$ is equivalent to $\left(\tilde{\pi} \times U_{\pi}, W_{\pi}\right)$ for some representation $\pi$ of $A$.

Proof. This follows from Green's imprimitivity theorem [3, Theorem 6] by checking that the inverse of the isomorphism of Proposition 3.3 transforms the induced representation of the imprimitivity algebra $\left(A \otimes C_{0}(G / N)\right) \tilde{\times} G$ into the representation $\tilde{\pi} \times U_{\pi} \times W_{\pi}$ of $A \widetilde{\times} G \times(G / N)^{-}$.

COROLLARY 3.5. If $\pi$ is a faithful representation of $A$, then $\left(\tilde{\pi} \times U_{\pi}, W_{\pi}\right)$ is a weakly universal covariant representation of $(A \tilde{\times} G, G / N, \widehat{\alpha})$.

Proof. This follows from the fact that a faithful representation induces a faithful representation of the imprimitivity algebra.

Now assume that $c: G / N \rightarrow G$ is a measurable cross section such that $c(N)=e$, and let $A$ be faithfully represented on $\mathscr{H}$. Denote $\mathscr{K}\left(L^{2}(G / N)\right)$ simply by $\mathscr{K}$.

THEOREM 3.6. There is an isomorphism of $A \widetilde{\times} G \times(G / N)^{\wedge}$ with $A \otimes \mathscr{K}$ which transforms the double dual action $\widehat{\hat{\alpha}}$ into the action $\widetilde{\alpha}$ of $G / N$ on $A \otimes \mathscr{K}$ defined by

$$
\widetilde{\alpha}_{s N}=\operatorname{Ad}\left(\left(1 \otimes \rho_{G / N}(s N)\right) v(s N, \cdot)\right) \circ \alpha_{c(s N)} \otimes \imath,
$$

where

$$
v(s N, t N)=\tau\left(c(s N) c\left(t^{-1} N\right) c\left(s t^{-1} N\right)^{-1}\right)^{*}
$$


Proof. Green [4] gives a discussion which, adapted to our context, shows that the (faithful) representation of the imprimitivity algebra $A \widetilde{\times}(G / N)^{\text {- }}$ induced from the identity representation of $A$ on $\mathscr{H}$ can be realized on $\mathscr{H} \otimes L^{2}(G / N)$ as $\pi_{c} \times U_{c} \times W_{c}$, where for $\xi \in \mathscr{H} \otimes L^{2}(G / N), t \in G$

$$
\begin{gathered}
{\left[\pi_{c}(a) \xi\right](t N)=\alpha_{c(t N)}^{-1}(a) \xi(t N), \quad a \in A,} \\
{\left[U_{c}(s) \xi\right](t N)=\tau\left(c(t N)^{-1} s c\left(s^{-1} t N\right)\right) \xi\left(s^{-1} t N\right), \quad s \in G,} \\
{\left[W_{c}(\phi) \xi\right](t N)=\phi(t N) \xi(t N), \quad \phi \in C_{0}(G / N),}
\end{gathered}
$$

and that moreover the image of $\pi_{c} \times U_{c} \times W_{c}$ is $A \otimes \mathscr{K}$. This is not quite the representation we require, however. Define

$$
u(s N, t N)=v\left(s N, t^{-1} N\right)^{*}, \quad s, t \in G .
$$

It is routine to verify that $(\alpha \circ c, u)$ is a twisted action of $G / N$ on $A$ in the sense of Busby and Smith [1], that is, $\alpha \circ c: G / N \rightarrow$ Aut $A$ is strongly measurable, $u: G / N \times G / N \rightarrow U(M(A))$ is strictly measurable, and for $r, s, t \in G$

$$
\begin{gathered}
\alpha_{c(N)}=l, \quad u(s N, N)=u(N, s N)=e, \\
\alpha_{c(s N)} \alpha_{c(t N)}=\operatorname{Ad} u(s N, t N) \circ \alpha_{c(s t N)}, \\
\alpha_{c(r N)} \circ u(s N, t N) u(r N, s t N)=u(r N, s N) u(r s N, t N) .
\end{gathered}
$$

Define a unitary operator $T$ on $\mathscr{H} \otimes L^{2}(G / N)$ by

$$
[T \xi](t N)=u\left(t^{-1} N, t N\right) \xi(t N) .
$$

The representation of $A \widetilde{\times} G \times(G / N)^{\wedge}$ which we require is

$$
\pi \times U \times W=\operatorname{Ad} T \circ\left(\pi_{c} \times U_{c} \times W_{c}\right) .
$$

Perhaps the easiest way to see that Ad $T$ leaves $A \otimes \mathscr{K}$ invariant is to use the observation of Packer and Raeburn [9, Lemma 3.5] to the effect that $A \otimes \mathscr{K}$ is the closure of $\left\{K_{\phi}: \phi \in L^{2}(G / N \times G / N, A)\right\}$, where

$$
\left[K_{\phi} \xi\right](s N)=\int_{G / N} \phi(s N, t N) \xi(t N) d(t N) .
$$

Packer and Raeburn impose separability restrictions which are not needed for this result.

We determine the representations $\pi, U$, and $W$. For $\pi$ we have

$$
\begin{aligned}
{[\pi(a) \xi](t N) } & =\left[\operatorname{Ad} T \circ \pi_{c}(a) \xi\right](t N)=u\left(t^{-1} N, t N\right)\left[\pi_{c}(a) T^{*} \xi\right](t N) \\
& =u\left(t^{-1} N, t N\right) \alpha_{c(t N)}^{-1}(a)\left[T^{*} \xi\right](t N) \\
& =\operatorname{Ad} u\left(t^{-1} N, t N\right) \circ \alpha_{c(t N)}^{-1}(a) \xi(t N)=\alpha_{c\left(t^{-1} N\right)}(a) \xi(t N) .
\end{aligned}
$$


For $U$, since

$$
U(s)=U\left(s c(s N)^{-1}\right) U \circ c(s N)=\pi \circ \tau\left(s c(s N)^{-1}\right) U \circ c(s N),
$$

we need only determine $U \circ c$. We have

$$
\begin{aligned}
{\left[U_{c} \circ c(s N) \xi\right](t N) } & =\tau\left(c(t N)^{-1} c(s N) c\left(c(s N)^{-1} t N\right)\right) \xi\left(c(s N)^{-1} t N\right) \\
& =\tau\left(c(t N)^{-1} c(s N) c\left(s^{-1} t N\right)\right) \xi\left(s^{-1} t N\right) \\
& =\tau \circ \operatorname{Ad} c(t N)^{-1}\left(c(s N) c\left(s^{-1} t N\right)\left(c(t N)^{-1}\right) \xi\left(s^{-1} t N\right)\right. \\
& =\alpha_{c(t N)}^{-1} \circ u\left(s N, s^{-1} t N\right) \xi\left(s^{-1} t N\right),
\end{aligned}
$$

so

$$
\begin{aligned}
{[U \circ c(s N) \xi](t N)=} & {\left[\operatorname{Ad} T \circ U_{c} \circ c(s N) \xi\right](t N) } \\
= & u\left(t^{-1} N, t N\right)\left[U_{c} \circ c(s N) T^{*} \xi\right](t N) \\
= & u\left(t^{-1} N, t N\right) \alpha_{c(t N)}^{-1} \circ u\left(s N, s^{-1} t N\right)\left[T^{*} \xi\right]\left(s^{-1} t N\right) \\
= & \alpha_{c\left(t^{-1} N\right)} \circ u\left(s N, s^{-1} t N\right) \\
& \times u\left(t^{-1} N, t N\right) u\left(t^{-1} s N, s^{-1} t N\right)^{*} \xi\left(s^{-1} t N\right) \\
= & u\left(t^{-1} N, s N\right) \xi\left(s^{-1} t N\right) .
\end{aligned}
$$

Of course, for $W$ we have

$$
\begin{aligned}
{[W(\phi) \xi](t N) } & =\left[\operatorname{Ad} T \circ W_{c}(\phi) \xi\right](t N) \\
& =\operatorname{Ad} u\left(t^{-1} N, t N\right)(\phi(t N)) \xi(t N)=\phi(t N) \xi(t N) .
\end{aligned}
$$

Now, by Definition 2.9 the double dual action $\widehat{\widehat{\alpha}}$ of $G / N$ on $A \tilde{\times} G \times(G / N)^{-}$ may be characterized in terms of the faithful representation $\pi \times U \times W$ by

$$
\widehat{\widehat{\alpha}}_{s N} \circ(\pi \times U)=\pi \times U, \quad \widehat{\widehat{\alpha}}_{s N} \circ W=W \circ \beta_{s N}^{G / N} .
$$

Therefore, we can conclude the proof by showing that $\tilde{\alpha}$ as defined in the statement of the theorem has these properties. For $\pi$,

$\left[\left(1 \otimes \rho_{G / N}(s N)\right) v(s N, \cdot) \alpha_{c(s N)} \otimes \imath \circ \pi(a) \xi\right](t N)$

$$
\begin{aligned}
& =\Delta_{G / N}(s N)^{1 / 2}\left[v(s N, \cdot) \alpha_{c(s N)} \otimes \imath \circ \pi(a) \xi\right](t s N) \\
& =\Delta_{G / N}(s N)^{1 / 2} u\left(s N, s^{-1} t^{-1} N\right)^{*}\left[\alpha_{c(s N)} \otimes \imath \circ \pi(a) \xi\right](t s N) \\
& =\Delta_{G / N}(s N)^{1 / 2} u\left(s N, s^{-1} t^{-1} N\right)^{*} \alpha_{c(s N)} \alpha_{c\left(s^{-1} t^{-1} N\right)}(a) \xi(t s N) \\
& =\Delta_{G / N}(s N)^{1 / 2} \alpha_{c\left(t^{-1} N\right)}(a) u\left(s N, s^{-1} t^{-1} N\right)^{*} \xi(t s N) \\
& =\Delta_{G / N}(s N)^{1 / 2} \alpha_{c\left(t^{-1} N\right)}(a)[v(s N, \cdot) \xi](t s N) \\
& =\alpha_{c\left(t^{-1} N\right)}(a)\left[\left(1 \otimes \rho_{G / N}(s N)\right) v(s N, \cdot) \xi\right](t s N) \\
& =\left[\pi(a)\left(1 \otimes \rho_{G / N}(s N)\right) v(s N, \cdot) \xi\right](t s N) .
\end{aligned}
$$


For $U$, it suffices to check $U \circ c$ :

$$
\begin{aligned}
{[(1 \otimes} & \left.\left.\rho_{G / N}(s N)\right) v(s N, \cdot) \alpha_{c(s N)} \otimes \imath \circ U \circ c(r N) \xi\right](t N) \\
& =\Delta_{G / N}(s N)^{1 / 2}\left[v(s N, \cdot) \alpha_{c(s N)} \otimes l \circ U \circ c(r N) \xi\right](t s N) \\
& =\Delta_{G / N}(s N)^{1 / 2} u\left(s N, s^{-1} t^{-1} N\right)^{*}\left[\alpha_{c(s N)} \otimes \imath \circ U \circ c(r N) \xi\right](t s N) \\
& =\Delta_{G / N}(s N)^{1 / 2} u\left(s N, s^{-1} t^{-1} N\right)^{*} \alpha_{c(s N)}\left(u\left(s^{-1} t^{-1} N, r N\right)\right) \xi\left(r^{-1} t s N\right) \\
& =\Delta_{G / N}(s N)^{1 / 2} u\left(t^{-1} N, r N\right) u\left(s N, s^{-1} t^{-1} r N\right)^{*} \xi\left(r^{-1} t s N\right) \\
& =\Delta_{G / N}(s N)^{1 / 2} u\left(t^{-1} N, r N\right)[v(s N, \cdot) \xi]\left(r^{-1} t s N\right) \\
& =u\left(t^{-1} N, r N\right)\left[\left(1 \otimes \rho_{G / N}(s N)\right) v(s N, \cdot) \xi\right]\left(r^{-1} t N\right) \\
& =\left[U \circ c(r N)\left(1 \otimes \rho_{G / N}(s N)\right) v(s N, \cdot) \xi\right](t N) .
\end{aligned}
$$

Finally, for $W$ we have

$$
\begin{aligned}
{[\operatorname{Ad}(} & \left.\left.\left(1 \otimes \rho_{G / N}(s N)\right) v(s N, \cdot)\right) \circ \alpha_{c(s N)} \otimes \imath \circ W(\phi) \xi\right](t N) \\
& =\left[\operatorname{Ad}\left(1 \otimes \rho_{G / N}(s N) \circ W(\phi) \xi\right](t N)\right. \\
& =\phi(t s N) \xi(t N)=\beta_{s N}^{G / N}(\phi)(t N) \xi(t N)
\end{aligned}
$$

Immediately after Proposition 3.3 we could have appealed to [4, Corollary 2.12] to show that

$$
A \tilde{\times} G \times(G / N)^{\wedge} \cong A \otimes \mathscr{K},
$$

but it would not have been as easy to obtain the double dual action. Also, Corollary 3.4 essentially shows that the dual crossed products of both the full and the reduced twisted crossed products are the same. Therefore, we could have deduced the full twisted crossed product duality from the reduced twisted crossed product duality of [11]. However, this would have necessitated a discussion of the relation between the twisted crossed products of Green and of Busby and Smith [1]. Specifically, in the presence of a measurable cross section every Green twisted crossed product is a Busby-Smith twisted crossed product, as is shown in the separable case in [9]. Again this would have been less efficient than the approach we took. However, if we had an imprimitivity theorem for induced representations of Busby-Smith twisted crossed products, we would have the corresponding full twisted crossed product duality; as we have no application in mind involving these more general twisted crossed products, we did not attempt to prove such an imprimitivity theorem.

We return to the discussion begun at the end of Section 2 regarding the failure of full crossed product duality for coactions. First we show that full duality does hold for dual coactions on full crossed products. What follows 
can in fact be done for twisted crossed products with no more difficulty, but for ease of writing we eschew the twist since we merely wish to make a point concerning duality.

THEOREM 3.7. If $A$ is a full crossed product by $G$, equipped with the dual coaction, then

$$
A \times \widehat{G} \times G=A \otimes \mathscr{K}
$$

Proof. Let $A$ be the crossed product of a $C^{*}$-algebra $B$ by an action $\alpha$ of $G$. Then by Theorem 3.6 the double dual action on $A \times \widehat{G}$ is conjugate to the action $\tilde{\alpha}$ on $B \otimes \mathscr{H}$. Further, as Packer and Raeburn observe [9], this latter action is outer conjugate to $\alpha \otimes l$, so we have

$$
A \times \widehat{G} \times G \cong B \otimes \mathscr{K} \times_{\tilde{\alpha}} G \cong B \otimes \mathscr{K} \times_{\alpha \otimes l} G \cong B \times_{\alpha} G \otimes \mathscr{K}=A \otimes \mathscr{K} .
$$

We present a contrasting result for reduced crossed products. First note that by Corollary 3.5 the dual coaction on a full crossed product by an action factors through a coaction, which we continue to refer to as "dual", on the reduced crossed product.

THEOREM 3.8. If $A$ is a reduced crossed product by $G$, equipped with the dual coaction, then

$$
A \times \widehat{G} \times{ }_{r} G=A \otimes \mathscr{K} .
$$

Proof. By Corollary 3.4, $A$ is faithfully represented in the multiplier algebra of $A \times \widehat{G}$. Hence, by Proposition 2.8(ii), the proposition to be proven is just Katayama's duality theorem [5, Theorem 8].

The preceding two results show that one can sometimes get $A \otimes \mathscr{K}$ from $A \times \widehat{G}$ using the full dual crossed product, and sometimes using the reduced dual crossed product. We wish to present an example, involving a direct sum of these two situations, where neither the full nor the reduced dual crossed product of $A \times \widehat{G}$ will yield $A \otimes \mathscr{K}$. First we must discuss direct sums of coactions. The following result is well-known.

LemmA 3.9. Let $A_{1}, A_{2}$, and $B$ be $C^{*}$-algebras. Then there is an isomorphism $\Phi_{B}^{A_{1}, A_{2}}$ of $\left(A_{1} \otimes B\right) \oplus\left(A_{2} \otimes B\right)$ onto $\left(A_{1} \oplus A_{2}\right) \otimes B$ determined by

$$
\Phi_{B}^{A_{1}, A_{2}}\left(a_{1} \otimes b, a_{1} \otimes b^{\prime}\right)=\left(a_{1}, 0\right) \otimes b+\left(0, a_{2}\right) \otimes b^{\prime} .
$$

Moreover,

$$
\Phi_{B}^{A_{1}, A_{2}}\left(\widetilde{M}\left(A_{1} \otimes B\right) \oplus \widetilde{M}\left(A_{2} \otimes B\right)\right) \subset \widetilde{M}\left(\left(A_{1} \oplus A_{2}\right) \otimes B\right) .
$$


Definition 3.10. Let $\delta^{i}$ be a coaction of $G$ on $A_{i}(i=1,2)$. Then the associated direct sum coaction is the map

$$
\delta^{1} \tilde{\oplus} \delta^{2}=\Phi_{C^{*}(G)}^{A_{1}, A_{2}} \circ\left(\delta^{1} \oplus \delta^{2}\right),
$$

where $\delta^{1} \oplus \delta^{2}$ is the usual direct sum of $\delta^{1}$ and $\delta^{2}$ as linear maps.

Proposition 3.11. If $\delta^{i}$ is a coaction of $G$ on $A_{i}(i=1,2)$, then $\delta^{1} \tilde{\oplus} \delta^{2}$ is a coaction of $G$ on $A_{1} \oplus A_{1}$. Moreover, $\delta^{1} \widetilde{\oplus} \delta^{2}$ is nondegenerate if both $\delta^{1}$ and $\delta^{2}$ are.

Proof. $\delta^{1} \widetilde{\oplus} \delta^{2}$ is clearly a strict monomorphism of $A_{1} \oplus A_{2}$ into $\widetilde{M}\left(\left(A_{1} \oplus\right.\right.$ $\left.\left.A_{2}\right) \otimes C^{*}(G)\right)$. By symmetry, it suffices to check the coaction identity on an element of the form $(a, 0)$ with $a \in A_{1}$. We have

$$
\left(\delta^{1} \tilde{\oplus} \delta^{2}\right) \otimes l \circ\left(\delta^{1} \tilde{\oplus} \delta^{2}\right)(a, 0)=\left(\delta^{1} \tilde{\oplus} \delta^{2}\right) \otimes l \circ \Phi_{C^{*}(G)}^{A_{1}, A_{2}}\left(\delta^{1}(a), 0\right) .
$$

We claim that

$$
\left(\delta^{1} \tilde{\oplus} \delta^{2}\right) \otimes \imath \circ \Phi_{C^{*}(G)}^{A_{1}, A_{2}}=\Phi_{C^{*}(G) \otimes C^{*}(G)}^{A_{1}, A_{2}} \circ\left(\left(\delta^{1} \otimes l\right) \oplus\left(\delta^{2} \otimes l\right)\right)
$$

on $\left(A_{1} \otimes C^{*}(G)\right) \oplus\left(A_{2} \otimes C^{*}(G)\right)$, and again by symmetry it suffices to check it on an element of the form $(b \otimes c, 0)$ with $b \in A_{1}, c \in C^{*}(G)$. On the one hand

$\left(\delta^{1} \tilde{\oplus} \delta^{2}\right) \otimes \imath \circ \Phi_{C^{*}(G)}^{A_{1}, A_{2}}(b \otimes c, 0)=\left(\delta^{1} \widetilde{\oplus} \delta^{2}\right) \otimes l((b, 0) \otimes c)=\Phi_{C^{*}(G)}^{A_{1}, A_{2}}\left(\delta^{1}(b), 0\right) \otimes c$, while on the other hand

$$
\Phi_{C^{*}(G) \otimes C^{*}(G)}^{A_{1}, A_{2}} \circ\left(\left(\delta^{1} \otimes i\right) \oplus\left(\delta^{2} \otimes i\right)\right)(b \otimes c, 0)=\Phi_{C^{*}(G) \otimes C^{*}(G)}^{A_{1}, A_{2}}\left(\delta^{1}(b) \otimes c, 0\right),
$$

so we can conclude the verification of the claim by noting that for $b^{\prime} \in A_{1}$, $c^{\prime} \in C^{*}(G)$ we have

$$
\Phi_{C^{*}(G)}^{A_{1}, A_{2}}\left(b^{\prime} \otimes c^{\prime}, 0\right) \otimes c=\left(b^{\prime}, 0\right) \otimes c^{\prime} \otimes c=\Phi_{C^{*}(G) \otimes C^{*}(G)}^{A_{1}, A_{2}}\left(b^{\prime} \otimes c^{\prime} \otimes c, 0\right) .
$$

Hence,

$$
\begin{aligned}
\left(\delta^{1} \widetilde{\oplus}\right. & \left.\delta^{2}\right) \otimes l \circ\left(\delta^{1} \widetilde{\oplus} \delta^{2}\right)(a, 0) \\
& =\Phi_{C^{*}(G) \otimes C^{*}(G)}^{A_{1}, A_{2}} \circ\left(\left(\delta^{1} \otimes l\right) \oplus\left(\delta^{2} \otimes l\right)\right)\left(\delta^{1}(a), 0\right) \\
& =\Phi_{C^{*}(G) \otimes C^{*}(G)}^{A_{1}, A_{2}}\left(\delta^{1} \otimes l \circ \delta^{1}(a), 0\right) \\
& =\Phi_{C^{*}(G) \otimes C^{*}(G)}^{A_{1}, A_{2}}\left(l \otimes \delta^{G} \circ \delta^{1}(a), 0\right) \\
& =\Phi_{C^{*}(G) \otimes C^{*}(G)}^{A_{1}, A_{2}} \circ\left(\left(l \otimes \delta^{G}\right) \oplus\left(l \otimes \delta^{G}\right)\right)\left(\delta^{1}(a), 0\right) .
\end{aligned}
$$

Therefore, we can conclude the verification of the coaction identity by showing that

$$
\imath \otimes \delta^{G} \circ \Phi_{C^{*}(G)}^{A_{1}, A_{2}}=\Phi_{C^{*}(G) \otimes C^{*}(G)}^{A_{1}, A_{2}} \circ\left(\left(l \otimes \delta^{G}\right) \oplus\left(l \otimes \delta^{G}\right)\right)
$$


on $\left(A_{1} \otimes C^{*}(G)\right) \oplus\left(A_{2} \otimes C^{*}(G)\right)$, and as before it suffices to check it on $(b \otimes c, 0)$ with $b \in A_{1}, c \in C^{*}(G)$ :

$$
\begin{aligned}
l \otimes \delta^{G} \circ \Phi_{C^{*}(G)}^{A_{1}, A_{2}}(b \otimes c, 0)=\imath \otimes \delta^{G}((b, 0) \otimes c)=(b, 0) \otimes \delta^{G}(c) \\
=\Phi_{C^{*}(G) \otimes C^{*}(G)}^{A_{1}, A_{2}}\left(b \otimes \delta^{G}(c), 0\right) \\
=\Phi_{C^{*}(G) \otimes C^{*}(G)}^{A_{1}, A_{2}} \circ\left(\left(l \otimes \delta^{G}\right) \oplus\left(l \otimes \delta^{G}\right)\right)(b \otimes c, 0) .
\end{aligned}
$$

Finally, nondegeneracy is clear.

Proposition 3.12. If $\delta^{i}$ is a coaction of $G$ on $A_{i}(i=1,2)$, then

$$
\begin{gathered}
\left(A_{1} \oplus A_{2}\right) \times{ }_{\delta^{1} \widetilde{\oplus} \delta^{2}} G=\left(A_{1} \times{ }_{\delta^{1}} G\right) \oplus\left(A_{2} \times{ }_{\delta^{2}} G\right) ; \\
\left(A_{1} \oplus A_{2}\right) \times{ }_{\delta^{1} \widetilde{\oplus} \delta^{2}, r} G=\left(A_{1} \times{ }_{\delta^{1}, r} G\right) \oplus\left(A_{2} \times{ }_{\delta^{2}, r} G\right) .
\end{gathered}
$$

Proof. (i) Let $(\pi, W)$ be a covariant representation of $\left(A_{1} \oplus A_{2}, G, \delta^{1}\right.$ $\widetilde{\oplus} \delta^{2}$ ) on $\mathscr{H}$. Then without loss of generality we may assume that for $i=1,2$ there is a representation $\pi_{i}$ of $A_{i}$ on a Hilbert space $\mathscr{H}_{i}$ such that $\mathscr{H}=\mathscr{H}_{1} \oplus \mathscr{H}_{2}$ and

$$
\pi\left(a_{1}, a_{2}\right)=\pi_{1}\left(a_{1}\right) \oplus \pi_{2}\left(a_{2}\right), \quad a_{i} \in A_{i} .
$$

We show that $\mathscr{H}_{1} \oplus\{0\}$ reduces $W$, whence for $i=1,2$ there is a representation $W_{i}$ of $C_{0}(G)$ on $\mathscr{H}_{i}$ such that

$$
W(\phi)=W_{1}(\phi) \oplus W_{2}(\phi), \quad \phi \in C_{0}(G) .
$$

Let $p$ denote the projection of $\mathscr{H}$ onto $\mathscr{H}_{1} \oplus\{0\}$, and let $\phi \in B(G)$. We have

$$
\begin{aligned}
W(\phi) p & =W(\phi) \pi(1,0)=S_{\phi}(W) \pi(1,0)=S_{\phi}(W(\pi(1,0) \otimes 1)) \\
& =S_{\phi}\left(\pi \otimes \imath \circ\left(\delta^{1} \widetilde{\oplus} \delta^{2}\right)(1,0) W\right)=S_{\phi}\left(\pi \otimes \imath \circ \Phi_{C^{*}(G)}^{A_{1}, A_{2}}\left(\delta^{1}(1), 0\right) W\right) .
\end{aligned}
$$

We claim that

$$
\pi \otimes \imath \circ \Phi_{C^{*}(G)}^{A_{1}, A_{2}}=\Phi_{C^{*}(G)}^{\pi_{1}\left(A_{1}\right), \pi_{2}\left(A_{2}\right)} \circ\left(\left(\pi_{1} \otimes \imath\right) \oplus\left(\pi_{2} \otimes \imath\right)\right)
$$

on $\left(A_{1} \otimes C^{*}(G)\right) \oplus\left(A_{2} \otimes C^{*}(G)\right)$, and as before it suffices to check this on an element of the form $(a \otimes c, 0)$ with $a \in A_{1}, c \in C^{*}(G)$ :

$$
\begin{aligned}
\pi \otimes l & \circ \Phi_{C^{*}(G)}^{A_{1}, A_{2}}(a \otimes c, 0)=\pi \otimes l((a, 0) \otimes c)=\left(\pi_{1}(a), 0\right) \otimes c \\
= & \Phi_{C^{*}(G)}^{\pi_{1}\left(A_{1}\right), \pi_{2}\left(A_{2}\right)}\left(\pi_{1}(a) \otimes c, 0\right) \\
= & \Phi_{C^{*}(G)}^{\pi_{1}\left(A_{1}\right), \pi_{2}\left(A_{2}\right)} \circ\left(\left(\pi_{1} \otimes l\right) \oplus\left(\pi_{2} \otimes l\right)\right)(a \otimes c, 0),
\end{aligned}
$$


establishing the claim. Therefore,

$$
\begin{aligned}
W(\phi) p & =S_{\phi}\left(\Phi_{C^{*}(G)}^{\pi_{1}\left(A_{1}\right), \pi_{2}\left(A_{2}\right)} \circ\left(\left(\pi_{1} \otimes l\right) \oplus\left(\pi_{2} \otimes l\right)\right)\left(\delta^{1}(1), 0\right) W\right) \\
& =S_{\phi}\left(\Phi_{C^{*}(G)}^{\pi_{1}\left(A_{1}\right), \pi_{2}\left(A_{2}\right)}(1 \otimes 1,0) W\right)=S_{\phi}((p \otimes 1) W)=p S_{\phi}(W)=p W(\phi),
\end{aligned}
$$

showing that $\mathscr{H}_{1} \oplus\{0\}$ indeed reduces $W$, so that we have representations $W_{1}$ and $W_{2}$ as described above.

We next show that $\left(\pi_{i}, W_{i}\right)$ is a covariant representation of $\left(A_{i}, G, \delta^{i}\right)$ $(i=1,2)$. By symmetry it suffices to show it for $i=1$. Let

$$
\Phi=\Phi_{C^{*}(G)}^{C^{*}\left(\pi_{1}, W_{1}\right), C^{*}\left(\pi_{2}, W_{2}\right)}
$$

For $a \in A_{1}$ we have

$$
\begin{aligned}
\Phi\left(\pi_{1}\right. & \left.\otimes \imath \circ \delta^{1}(a) W_{1}, 0\right) \\
& =\Phi\left(\left(\pi_{1} \otimes \imath \circ \delta^{1}(a), 0\right)\left(W_{1}, W_{2}\right)\right)=\Phi\left(\pi_{1} \otimes \imath \circ \delta^{1}(a), 0\right) W \\
& =\Phi \circ\left(\left(\pi_{1} \otimes \imath\right) \oplus\left(\pi_{2} \otimes \imath\right)\right)\left(\delta^{1}(a), 0\right) W=\left(\left(\pi_{1} \oplus \pi_{2}\right) \otimes \imath \circ \Phi\left(\delta^{1}(a), 0\right)\right) W \\
& =\left(\left(\pi_{1} \oplus \pi_{2}\right) \otimes \imath \circ\left(\delta^{1} \widetilde{\oplus} \delta^{2}\right)(a, 0)\right) W=W\left(\left(\pi_{1} \oplus \pi_{2}\right)(a, 0) \otimes 1\right) \\
& =W\left(\left(\pi_{1}(a), 0\right) \otimes 1\right)=\Phi\left(W_{1}, W_{2}\right)\left(\left(\pi_{1}(a), 0\right) \otimes 1\right) \\
& =\Phi\left(\left(W_{1}, W_{2}\right)\left(\pi_{1}(a) \otimes 1,0\right)\right)=\Phi\left(W_{1}\left(\pi_{1}(a) \otimes 1\right), 0\right),
\end{aligned}
$$

and therefore

$$
W_{1} \in \operatorname{Hom}\left(\pi_{1} \otimes 1, \pi_{1} \otimes \imath \circ \delta^{1}\right),
$$

since $\Phi$ is injective.

It is now clear that

$$
C^{*}(\pi, W)=C^{*}\left(\pi_{1}, W_{1}\right) \oplus C^{*}\left(\pi_{2}, W_{2}\right) .
$$

Conversely, every pair of covariant representations of $\left(A_{1}, G, \delta^{1}\right)$ and $\left(A_{2}, G, \delta^{2}\right)$ arises in this manner, so part (i) is proven.

For part (ii), it is not difficult to check that if $(\pi, W)$ is the regular covariant representation of $\left(A_{1} \oplus A_{2}, G, \delta^{1} \widetilde{\oplus} \delta^{2}\right)$, then, for $i=1,2,\left(\pi_{i}, W_{i}\right)$ will be the regular covariant representation of $\left(A_{i}, G, \delta^{i}\right)$.

Now we have

$$
\begin{aligned}
& \left(C^{*}(G) \oplus C_{r}^{*}(G)\right) \times \widehat{G} \times G=\left(\left(C^{*}(G) \times \widehat{G}\right) \oplus\left(C_{r}^{*}(G) \times \widehat{G}\right)\right) \times G \\
& \quad=\left(C^{*}(G) \times \widehat{G} \times G\right) \oplus\left(C_{r}^{*}(G) \times \widehat{G} \times G\right)=\left(C^{*}(G) \otimes \mathscr{K}\right) \oplus\left(C^{*}(G) \otimes \mathscr{K}\right) \\
& \quad \cong\left(C^{*}(G) \oplus C^{*}(G)\right) \otimes \mathscr{K},
\end{aligned}
$$

while similarly

$$
\left(C^{*}(G) \oplus C_{r}^{*}(G)\right) \times \widehat{G} \times_{r} G \cong\left(C_{r}^{*}(G) \oplus C_{r}^{*}(G)\right) \otimes \mathscr{K} .
$$


Hence, neither dual crossed product yields

$$
\left(C^{*}(G) \oplus C_{r}^{*}(G)\right) \otimes \mathscr{K} .
$$

However, it is easy in this example to construct a representation of $\left(C^{*}(G) \oplus\right.$ $\left.C_{r}^{*}(G)\right) \times \widehat{G} \times G$ whose image is $\left(C^{*}(G) \oplus C_{r}^{*}(G)\right) \otimes \mathscr{K}$. We do not know whether it is always possible to construct a representation of $A \times \widehat{G} \times G$ whose image is $A \otimes \mathscr{K}$.

\section{References}

[1] R. C. Busby and H. A. Smith, 'Representations of twisted group algebras,' Trans. Amer. Math. Soc. 132 (1968), 79-99.

[2] E. C. Gootman and A. J. Lazar, 'Applications of non-commutative duality to crossed product $C^{*}$-algebras determined by an action or coaction', preprint.

[3] P. Green, 'The local structure of twisted covariance algebras', Acta. Math. 140 (1978), 191-250.

[4] P. Green, 'The structure of imprimitivity algebras,' J. Funct. Anal. 36 (1980), 88-104.

[5] Y. Katayama, 'Takesaki's duality for a non-degenerate co-action,' Math. Scand. 55 (1985), 141-151.

[6] M. B. Landstad, J. Phillips, I. Raeburn, and C. E. Sutherland, 'Representations of crossed products by coactions and principal bundles,' Trans. Amer. Math. Soc. 299 (1987), 747-784.

[7] K. Mansfield, Induced representations of crossed products by coactions, (dissertation, Univ. N. S. W., Sydney, Australia).

[8] K. Mansfield, 'Induced representations of crossed products by coactions,' Proc. Cent. Math. Anal. Austral. Nat. Univ. 17 (1988), 191-196.

[9] J. A. Packer and I. Raeburn, 'Twisted crossed products of $C^{*}$-algebras,' preprint.

[10] G. K. Pedersen, $C^{*}$-algebras and their automorphism groups, (Academic Press, New York, 1979).

[11] J. C. Quigg, 'Duality for reduced twisted crossed products of $C^{*}$-algebras,' Indiana Univ. Math. J. 35 (1986), 549-571.

[12] I. Raeburn, 'A duality theorem for crossed products by nonabelian groups,' Proc. Cent. Math. Anal. Austral. Nat. Univ. 15 (1987), 214-227.

[13] M. A. Rieffel, 'Induced representations of $C^{*}$-algebras,' $A d v$. in Math. 13 (1974), 176257.

Department of Mathematics

Arizona State University

Tempe, Arizona 85287

U.S.A. 